06;12

\title{
Распределение температуры в образце с микроскопическим включением второй фазы при облучении низкоэнергетическим импульсным сильноточным электронным пучком
}

() Д.А. Шепель, А.Б. Марков

Институт сильноточной электроники СО РАН, Томск

IE-mail: almar@Ive.hcei.tsc.ru

Поступило в Редакцию 19 апреля 2016 г.

С помощью методов численного моделирования рассчитано температурное поле, возникающее в поверхностном слое мишени из никелида титана, содержащей включения интерметаллида $\mathrm{NiTi}_{2}$, облучаемой низкоэнергетическим сильноточным электронным пучком микросекундной длительности. Полученное температурное поле сравнивалось с температурным полем, рассчитанным ранее для мишени из нержавеющей стали $316 \mathrm{~L}$, содержащей включение из сульфида марганца $(\mathrm{MnS})$. Обнаружено, что, как и в случае с нержавеющей сталью, в области расположения включения существует перегрев. Однако величина перегрева в случае никелида титана $(12 \mathrm{~K})$ существенно ниже, чем в случае нержавеющей стали $316 \mathrm{~L}(283 \mathrm{~K})$. Также существенно отличается динамика плавления двух систем.

DOI: 10.21883/PJTF.2017.03.44221.16301

Нержавеющая сталь $316 \mathrm{~L}$ и никелид титана (NiTi) - металлические сплавы, которые, благодаря своим физическим и химическим свойствам, до сих пор широко применяются для изготовления имплантатов [1]. 
Одной из наиболее важных характеристик материалов с точки зрения применения в медицине является коррозионная стойкость, которая может быть существенно повышена путем облучения низкоэнергетическим сильноточным электронным пучком (НСЭП). Однако известно, что в результате обработки поверхности НСЭП на облучаемой поверхности возможно образование микрократеров. Появление микрократеров ухудшает свойства поверхности. Причиной их появления является присутствие в материале включений второй фазы. В частности, показано, что на облучаемой поверхности образца из нержавеющей стали местами возникновения микрократеров являются места расположения частиц сульфида марганца $(\mathrm{MnS})$. Для образцов из никелида титана характерно появление микрократеров на местах расположения частиц интерметаллида $\mathrm{NiTi}_{2}$ [2]. Поскольку материал включений второй фазы имеет теплофизические свойства, отличные от свойств материала матрицы, то его присутствие будет оказывать влияние на распределение температуры в области расположения включения. Знание распределения температуры необходимо для понимания механизма образования кратеров.

Цель данной работы заключалась в расчете и анализе температурных полей, индуцированных в образцах из нержавеющей стали $316 \mathrm{~L}$ и NiTi. Полагалось, что материалы являются гомогенными, но содержат микроскопические включения вторых фаз.

Двумерная расчетная область представляла собой квадрат со стороной $b=30 \mu \mathrm{m}$, в центре одной из сторон которого располагалось включение размером $1 \times 1 \mu \mathrm{m}$ с отличными от матрицы теплофизическими свойствами. Эта сторона квадрата нагревалась однородным электронным пучком. Координатные оси были выбраны таким образом, чтобы облучаемая поверхность располагалась на координатной оси $X$, а направление падения пучка совпадало с положительным направлением координатной оси $Y$.

Моделирование температурного поля проводилось путем решения двумерного нелинейного нестационарного уравнения теплопроводности, учитывающего процессы плавления и кристаллизации и имеющего следующий вид:

$$
\begin{aligned}
\rho(c(T) & \left.+\delta\left(T(x, y, t)-T_{m}\right) L_{m}\right) \frac{\partial T(x, y, t)}{\partial t}=\frac{\partial}{\partial x}\left(k(T) \frac{\partial T(x, y, t)}{\partial x}\right) \\
& +\frac{\partial}{\partial y}\left(k(T) \frac{\partial T(x, y, t)}{\partial y}\right)+W(x, y, t),
\end{aligned}
$$

Письма в ЖТФ, 2017, том 43, вып. 3 
с граничными условиями, отражающими теплоизолированность пластины:

$$
-\left.k(T) \frac{\partial T(x, y, t)}{\partial x}\right|_{x=0 ; b}=0, \quad-\left.k(T) \frac{\partial T(x, y, t)}{\partial y}\right|_{y=0 ; b}=0,
$$

и начальным условием $T(x, y, 0)=T_{0}$. Здесь $\rho$ - плотность материала; $c(T)$ - удельная теплоемкость; $\delta$ - дельта-функция Дирака; $T(x, y, t)$ - температурное поле; $T_{m}$ - температура плавления; $L_{m}-$ скрытая теплота плавления; $k(T)$ - теплопроводность; $t-$ время; $x$ и $y$ - пространственные координаты; $W(x, y, t)$ - функция тепловых источников, характеризующая плотность мощности источников тепла и вычисляемая с помощью известных полуэмпирических формул [3]. Заметим, однако, что область применимости алгоритма ограничивается случаем расположения соседних включений на расстоянии $s>b$, когда температурные процессы, протекающие в области разных включений, слабо влияют друг на друга. Что касается физической адекватности описываемого алгоритма, она подтверждается тем, что на основе рассчитанных ранее данных [4] авторам [5] удалось получить оценку скорости радиального распространения расплава, которая хорошо совпала с экспериментальными результатами и дала возможность обосновать механизм формирования рельефа поверхности после облучения электронным пучком.

Расчеты выполнялись для двух типов образцов: первый - матрица из никелида титана, содержащая в центре облучаемой поверхности включение из $\mathrm{NiTi}_{2}\left(\mathrm{NiTi} / \mathrm{NiTi}_{2}\right)$; второй - матрица из нержавеющей стали 316L с включением из сульфида марганца (SS 316L/MnS). Теплофизические свойства материалов матрицы и включения, используемые при расчетах, приведены в таблице. Они полагались одинаковыми для твердой и жидкой фазы.

Параметры пучка в данных расчетах подбирались таким образом, чтобы для обеих систем на облучаемой поверхности реализовывался режим начального плавления, характеризующийся толщиной расплава на периферии облучаемой поверхности $\sim 1 \mu \mathrm{m}$. Для $\mathrm{NiTi} / \mathrm{NiTi}_{2}$ режим начального плавления реализовывался при плотности вкладываемой энергии $2 \mathrm{~J} / \mathrm{cm}^{2}$, а для SS $316 \mathrm{~L} / \mathrm{MnS}$ - при $3 \mathrm{~J} / \mathrm{cm}^{2}$. При этом длительность импульса составляла $4 \mu \mathrm{s}$, а энергия электронов $-30 \mathrm{keV}$. Предполагается, что параметры пучка в процессе облучения остаются постоянными.

Письма в ЖТФ, 2017, том 43, вып. 3 
Теплофизические свойства материалов

\begin{tabular}{c|c|c|c|c|c}
\hline Материал & $\rho, \mathrm{kg} / \mathrm{m}^{3}$ & $k, \mathrm{~W} /(\mathrm{m} \cdot \mathrm{K})$ & $c, \mathrm{~J} /(\mathrm{kg} \cdot \mathrm{K})$ & $T_{m}, \mathrm{~K}$ & $L_{m}, \mathrm{~kJ} / \mathrm{kg}$ \\
\hline $\mathrm{NiTi}$ & $6.45 \cdot 10^{3}$ & 18 & 438.6 & 1583 & 318.8 \\
$\mathrm{NiTi}_{2}$ & $6 \cdot 10^{3}$ & 15 & 485.4 & 1288 & 406.74 \\
$\mathrm{SS} 316 \mathrm{~L}$ & $8 \cdot 10^{3}$ & 16.3 & 500 & 1693 & 247 \\
$\mathrm{MnS}$ & $4 \cdot 10^{3}$ & 2 & 582.8 & 1803 & 287
\end{tabular}

Путем численного моделирования были получены распределения температуры вдоль облучаемой поверхности и по глубине для двух видов образцов под воздействием НСЭП. На основе вычисления распределения температуры вдоль облучаемой поверхности были получены зависимости величины перегрева, под которым понималась разность между температурами в данной точке поверхности и на периферии образца, от координаты поверхности. Зависимости перегрева $(\Delta T)$ от координаты вдоль облучаемой поверхности для двух видов образцов представлены на рис. 1. Из характера зависимостей видно, что максимальное значение перегрева достигается в центре облучаемой поверхности, т.е. в области расположения включения. Кроме того, для двух систем значения перегрева существенно различаются. При облучении образца из никелида титана (кривая 1) перегрев, возникающий в центре мишени, составляет $12 \mathrm{~K}$, а для образца из нержавеющей стали $316 \mathrm{~L}$ (кривая 2) он составляет $283 \mathrm{~K}$, т. е. почти в 25 раз больше. Из таблицы теплофизических свойств материалов видно, что теплоемкость, коэффициент теплопроводности и плотность $\mathrm{NiTi}$ и $\mathrm{NiTi}_{2}$ различаются незначительно. В то время как коэффициент теплопроводности нержавеющей стали $316 \mathrm{~L}$ почти на порядок выше коэффициента теплопроводности сульфида марганца, что и вызывает заметный перегрев поверхности в области расположения включения.

Неоднородность распределения температуры как вдоль поверхности, так и по глубине означает наличие градиентов температуры. В случае NiTi градиент температуры вдоль облучаемой поверхности к концу импульса составлял $2.2 \cdot 10^{6} \mathrm{~K} / \mathrm{m}$, а по глубине образца $-1.4 \cdot 10^{8} \mathrm{~K} / \mathrm{m}$. При облучении образца из нержавеющей стали $316 \mathrm{~L}$ градиент температуры вдоль облучаемой поверхности составлял $5.1 \cdot 10^{7} \mathrm{~K} / \mathrm{m}$, а по глубине образца $-2.4 \cdot 10^{8} \mathrm{~K} / \mathrm{m}$. Видно, что для $\mathrm{NiTi} / \mathrm{NiTi}_{2}$ градиенты различаются между собой более чем в 60 раз, а для $\mathrm{SS} 316 \mathrm{~L} / \mathrm{MnS}$ всего

Письма в ЖТФ, 2017, том 43, вып. 3 


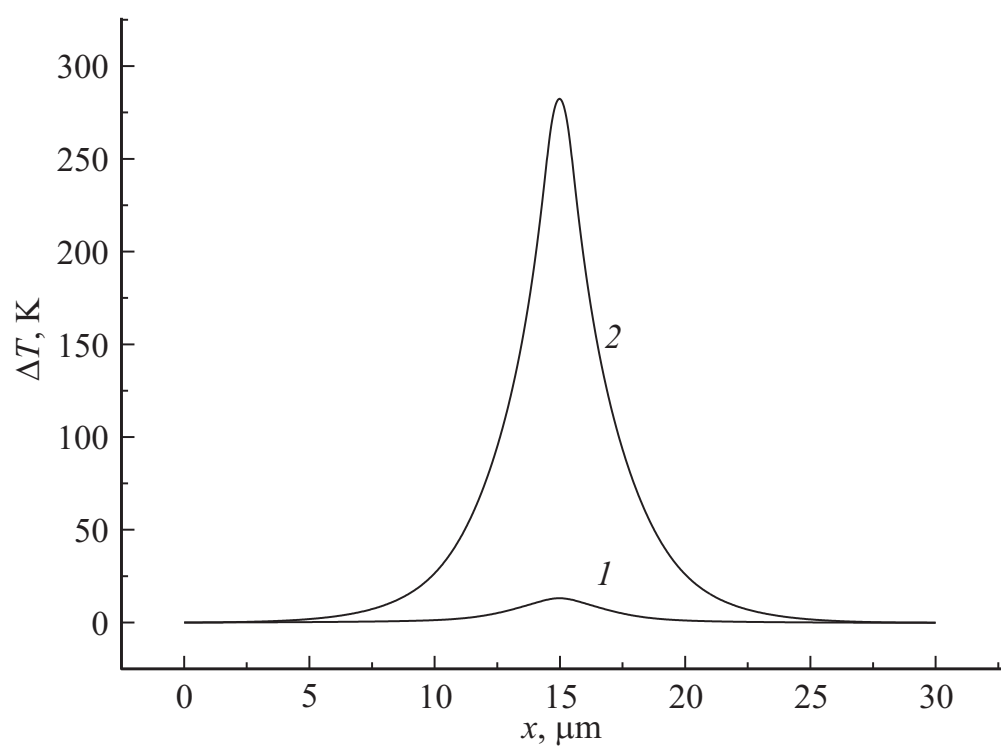

Рис. 1. Зависимость величины перегрева в центральной области поверхности, содержащей включение, относительно ее периферии: $1-\mathrm{NiTi} / \mathrm{NiTi}_{2}, 2-$ SS 316L/MnS.

в 5 раз. Кроме того, видно, что градиент температуры вдоль облучаемой поверхности для SS 316L/MnS превышает более чем в 20 раз значение градиента для образца $\mathrm{NiTi} / \mathrm{NiTi}_{2}$. Наличие градиентов температуры приводит к термокапиллярной конвекции, что, в свою очередь, является наиболее вероятной причиной возникновения кратеров на поверхности материалов [4-7].

Из литературы известно, что в результате облучения на поверхности образца из нержавеющей стали $316 \mathrm{~L}$ доля образующихся кратеров составляет 0.25 , это означает, что четыре включения дают один кратер [2]. На поверхности образца из никелида титана доля образующихся кратеров составляет приблизительно 0.07 , т.е. почти в 4 раза меньше, чем для стали. Следовательно, существует корреляция между величиной температурного градиента и вероятностью образования кратера на включении. Чем больше градиент вдоль поверхности, тем выше вероятность образования кратеров.

Письма в ЖТФ, 2017, том 43, вып. 3 


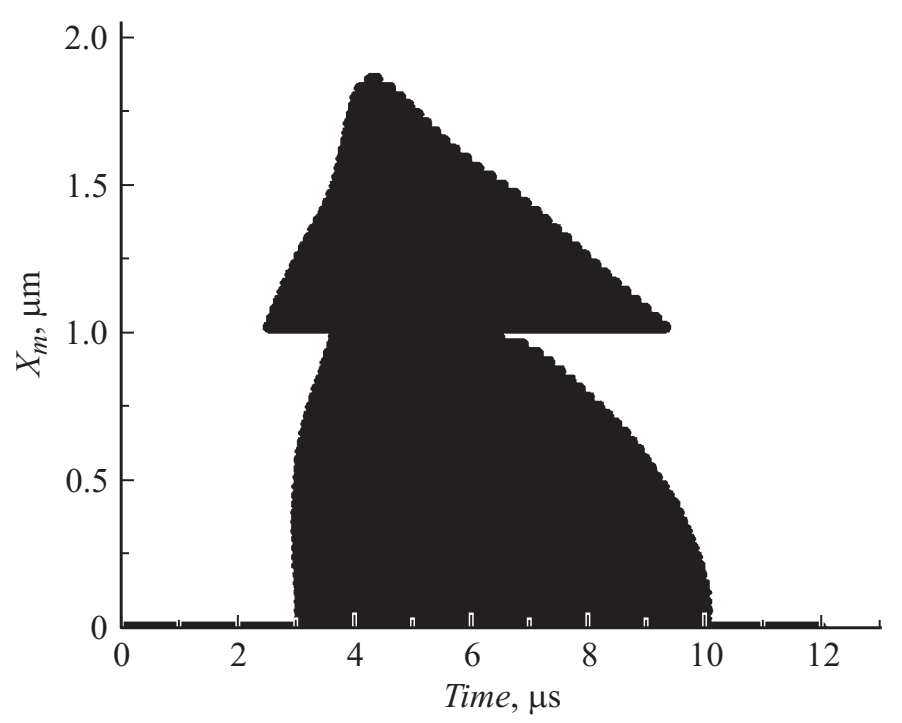

Рис. 2. Зависимость толщины расплава системы SS $316 \mathrm{~L} / \mathrm{MnS}$ в центре облучаемой поверхности в разные моменты времени.

Наличие на облучаемой поверхности включения, обладающего теплофизическими свойствами, отличными от теплофизических свойств материала матрицы, оказывает существенное влияние не только на величину возникающего перегрева, но и на процесс плавления. Плавление поверхности образца из нержавеющей стали $316 \mathrm{~L}$ с включением из $\mathrm{MnS}$ уже обсуждалось в [4]. Однако при более детальном рассмотрении оказывается, что процесс плавления начинается в материале матрицы за включением, что видно из рис. 2. На представленном графике закрашенная область соответствует толщине расплава в центре облучаемой поверхности в разные моменты времени. Видно, что первая порция расплава появляется на глубине около $1 \mu \mathrm{m}$. Затем, примерно через $1 \mu \mathrm{s}$, расплав появляется на облучаемой поверхности. Эти области расплавов разделены нерасплавленным материалом включения. К концу импульса расплав становится сплошным, а его толщина достигает почти $2 \mu \mathrm{m}$, затем начинается процесс кристаллизации материалов. Такое несплошное плавление материалов объясняется тем, что максимум

Письма в ЖТФ, 2017, том 43, вып. 3 

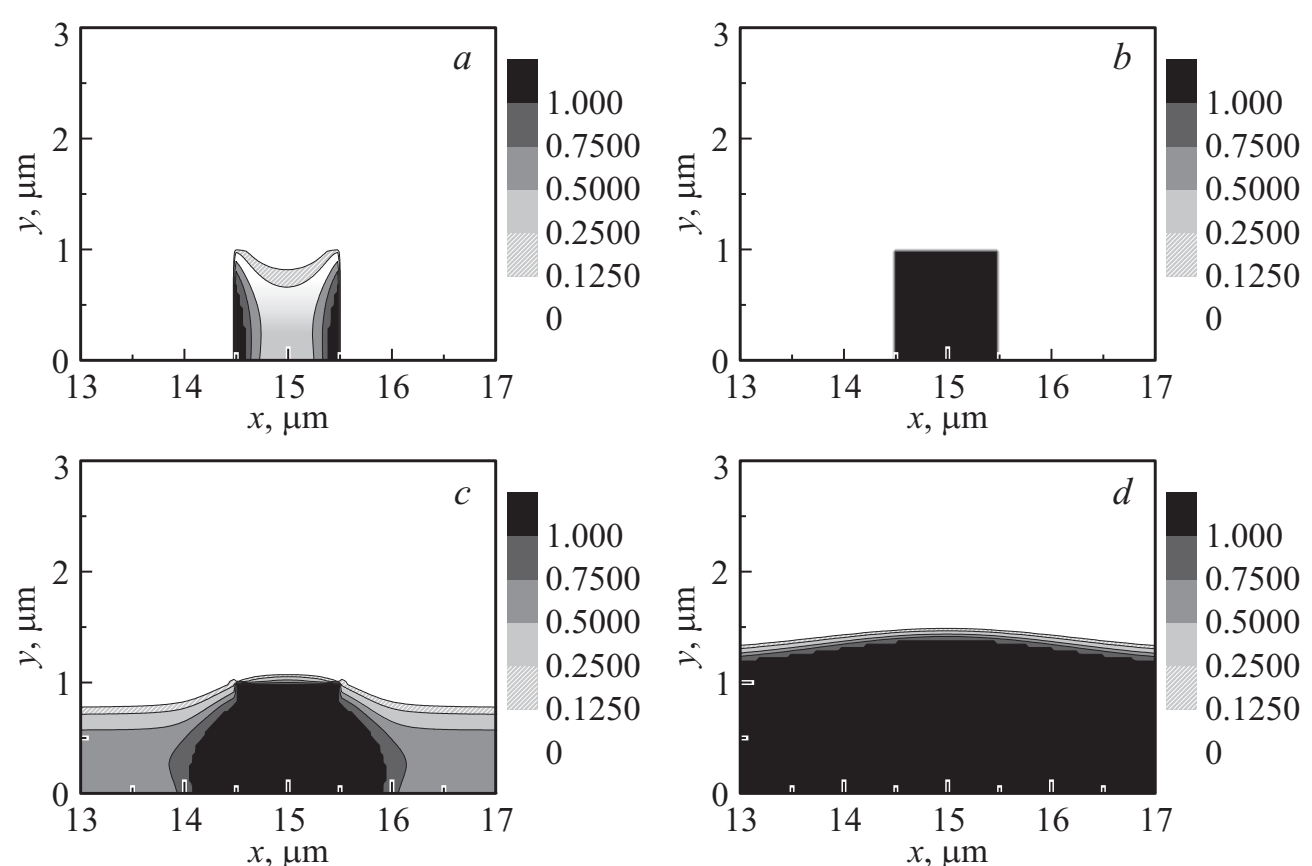

Рис. 3. Фазовые диаграммы для системы $\mathrm{NiTi} / \mathrm{NiTi}_{2}$ в моменты времени $2.0(a), 2.3(b), 3.3$ (c) и $4.0(d) \mu \mathrm{s}$ соответственно. Области твердой фазы, двухфазной зоны и расплава выделены белым, оттенками серого и черным цветом соответственно. НСЭП падает в положительном направлении оси у (снизу рисунка). 
энерговыделения располагается близко к тыльной стороне включения, а температура и скрытая теплота плавления сульфида марганца выше, чем для нержавеющей стали $316 \mathrm{~L}$.

На рис. 3 представлены фазовые диаграммы, демонстрирующие динамику процесса плавления $\mathrm{NiTi} / \mathrm{NiTi}_{2}$ под воздействием НСЭП. Цветовая шкала, приведенная сбоку от диаграммы, показывает долю вложенной скрытой теплоты плавления: белым цветом закрашены области, где процесс плавления еще не начался, а черным - области, где материал полностью расплавлен. Плавление образца из никелида титана начинается на границах включения примерно через $2 \mu$ s после начала облучения. Область расплава от границ включения распространяется к его центру (рис. 3,a). К моменту $2.3 \mu$ s включение уже полностью расплавлено (рис. $3, b$ ), а плавление материала матрицы (NiTi) начинается после $3 \mu \mathrm{s}$ (рис. 3,c). Затем область расплава быстро распространяется к периферии образца. К $3.6 \mu \mathrm{s}$ расплав толщиной около $0.6 \mu \mathrm{m}$ покрывает всю поверхность. Далее толщина расплавленного слоя на поверхности образца постепенно увеличивается и к моменту окончания импульса достигает $1.2 \mu \mathrm{m}$ на периферии и $1.4 \mu \mathrm{m}$ в центре облучаемой поверхности (рис. 3, $d$ ). До момента времени $4.8 \mu \mathrm{s}$ вся облучаемая поверхность покрыта расплавом. Примерно к $5.5 \mu \mathrm{s}$ материал матрицы полностью кристаллизуется, в то время как включение остается расплавленным. Кристаллизация включения начинается после $7.8 \mu \mathrm{s}$ и продолжается до момента $9.8 \mu \mathrm{s}$.

Из полученных результатов видно, что наличие включения на облучаемой поверхности приводит к неоднородности распределения температуры вдоль этой поверхности. Степень неоднородности распределения, характеризуемая перегревом, для разных систем может существенно отличаться: это объясняется соотношением теплофизических свойств материалов матрицы и включения. Существует корреляция между величиной градиента температуры и вероятностью образования кратера на включении. Чем больше градиент температуры вдоль поверхности, тем выше вероятность образования кратеров. Процесс плавления систем SS $316 \mathrm{~L} / \mathrm{MnS}$ и $\mathrm{NiTi} / \mathrm{NiTi}_{2}$ существенно отличается: плавление системы SS 316L/MnS начинается в приповерхностном слое, а не на облучаемой поверхности.

Исследование выполнено при финансовой поддержке РФФИ в рамках научного проекта № 16-08-00920 a.

Письма в ЖТФ, 2017, том 43, вып. 3 


\section{Список литературы}

[1] Hermawan H., Ramdan D., Djuansjah J.R.P. // Biomedical Engineering: from Theory to Applications / R. Fazel (ed.). InTech Pub, Croatia, 2011. P. 411-430.

[2] Zou J., Zhang K., Dong C. et al. // Appl. Phys. Lett. 2006. V. 86. P. 041913.

[3] Markov A.B., Rotshtein V.P. // Nucl. Instr. Meth. Phys. Res. B. 1997. V. 132. P. 79-86.

[4] Шепель Д.А., Марков А.Б. // Письма в ЖТФ. 2011. Т. 37. В. 16. С. 63-70.

[5] Озур Г.Е., Проскуровский Д.И., Ротштейн В.П. // Письма в ЖТФ. 2016. T. 42. B. 6. C. $97-103$.

[6] Марков А.Б., Мейснер Л.Л., Яковлев Е.В. и др. // Известия вузов. Физика. 2015. T. 58. № 9/3. C. 173-177.

[7] Meisner L.L., Markov A.B., Proskurovsky D.I. et al. // Surf. Coat. Tech. 2016. V. 302. P. 495-506.

Письма в ЖТФ, 2017, том 43, вып. 3 